\title{
Non-Diabetic Chronic Kidney Disease Influences Retinal Microvasculature
}

\author{
Marcus Baumann ${ }^{\text {a Sonja Schwarz }}{ }^{\mathrm{a}}$ Konstantin Kotliar ${ }^{\mathrm{b}}$ \\ Maximilian von Eynatten ${ }^{\mathrm{a}}$ Arno Schmidt Trucksaess ${ }^{\mathrm{d}}$ Klaus Burkhardt ${ }^{\mathrm{c}}$ \\ Jens Lutz ${ }^{a} \quad$ Uwe Heemann $^{\mathrm{a}}$ Ines Lanzl ${ }^{\mathrm{b}}$ \\ Departments of a Nephrology and ${ }^{b}$ Ophthalmology, Klinikum rechts der Isar, Technical University Munich, Munich, \\ and 'Nephrological Clinic, Weissenburg, Germany; ${ }^{\mathrm{I}}$ Institute of Exercise and Health Sciences, University of Basel, \\ Basel, Switzerland
}

\section{Key Words}

Retinal vessel analysis · Arteriolar-venular ratio $\cdot$ Arteriolar narrowing $\cdot$ Chronic kidney disease

\begin{abstract}
Background: Chronic kidney disease (CKD) is characterized by increased cerebrovascular risk. Retinal vessel analysis (RVA) is an accepted measure of the retinal microvasculature, mirrors hypertension and cardiovascular morbidity. Epidemiological studies demonstrate narrower retinal arterioles with declining renal function. The effect of CKD on the retinal microcirculation remains uncertain. Methods: RVA was performed in 34 non-diabetic CKD patients and 33 agematched volunteers with normal renal function. Retinal photographs were digitized, vascular lumen diameters measured and the ratio of retinal arteriolar and venular lumen diameters (AVR) calculated. Office blood pressure (BP) was measured and cardiovascular risk factors assessed. Results: AVR was lower in CKD patients as compared to age-matched volunteers $(0.77 \pm 0.05$ vs. $0.86 \pm 0.06 ; p<0.05)$. In particular, retinal arterioles were narrower in CKD patients as compared to volunteers $(169.6 \pm 20.4$ vs. $189.5 \pm 14.2 \mu \mathrm{m} ; \mathrm{p}<$ 0.01). In CKD, estimated glomerular filtration rate, $\mathrm{BP}$ and re-
\end{abstract}

nin-angiotensin system blocker independently predicted AVR. Moreover, retinal arteriolar diameter independently predicted renal function $(\beta=0.33 ; p<0.05)$. Conclusion: CKD narrowed retinal arterioles suggesting an extended effect of CKD on the cerebral microvasculature. This study shows that in CKD patients, renal function, BP status and renin-angiotensin system blockade independently predict AVR as a marker for microvascular damage and that retinal microvasculature can predict renal function.

Copyright $\odot 2009$ S. Karger AG, Basel

\section{Introduction}

Microcirculation is defined by precapillary arterioles and small arteries, that is, arteries with diameters $<350$ $\mu \mathrm{m}$ [1]. These vessels regulate the steep decline in blood pressure (BP) [2]. Vascular hypertrophy and arteriosclerotic changes in this part of the vasculature have been demonstrated to play a prognostic role for cardiovascular events [3-6]. This includes the prevalence and incidence of stroke. Moreover, retinal microvascular abnormalities are associated with cerebrovascular events [7]. Common abnormalities include microaneurysms, soft and hard

\section{KARGER}

Fax +41613061234 E-Mail karger@karger.ch www.karger.com
(C) 2009 S. Karger AG, Basel

$1420-4096 / 09 / 0326-0428 \$ 26.00 / 0$

Accessible online at:

www.karger.com/kbr
Dr. Marcus Baumann, $\mathrm{PhD}$

Department of Nephrology, Klinikum rechts der Isar, Technical University Munich Ismaninger Strasse 22, DE-81675 Munich (Germany)

Tel. +49894140 6704, Fax +498941404878

E-Mail marcus.baumann@lrz.tum.de 
exudates, hemorrhages and vessel tortuosity, beside others [8]. However, evidence is lacking that routine funduscopy is of additional value in the management of hypertensive patients [9].

Recently, non-invasive retinal vessel analysis (RVA) has received increased attention $[10,11]$. It is highly standardized by computer-assisted digital measurement of the ratio of retinal arteriolar and venular diameters (AVR) and has been evaluated in large-scale epidemiological studies [12-14]. Several of these studies additionally calculated renal function and observed that retinal microvascular abnormalities were associated with renal dysfunction $[15,16]$. However, no study confirmed these epidemiological results in a cohort with chronic kidney disease (CKD).

CKD patients are characterized by an increased cardiovascular mortality, including an elevated cerebrovascular risk [17]. Therefore, performing RVA in CKD patients may help in understanding the link between renal insufficiency and elevated cerebrovascular risk. In this respect, proteinuria as a marker of microcirculatory damage is of particular interest as proteinuria characterized not only the severity of CKD [18] but, in general population studies, was more prominent in subjects with arteriolar narrowing [16].

We hypothesized that CKD is accompanied by retinal microvascular narrowing and that in CKD patients, proteinuria is independently associated with AVR. To exclude microvascular effects associated with diabetes, we focused on non-diabetic subjects. To test our hypotheses, we performed RVA in non-diabetic CKD patients and age-matched controls with normal renal function.

\section{Methods}

In a cross-sectional study, $34 \mathrm{CKD}$ patients and 33 age-matched volunteers were included. Additionally, 10 hemodialysis patients were included for validation of the accuracy of retinal measurements. Patients and volunteers were recruited from the Klinikum rechts der Isar and the Nephrological Clinic Weissenburg. In all patients, RVA was performed and renal function and office BP were assessed. Research has been carried out in accordance with the Declaration of Helsinki (2000) of the World Medical Association and has been approved by the local Ethics Committee of the institution. Written informed consent was obtained from each patient after full explanation of the purpose, nature and risk of all procedures.

Glomerular filtration rate was estimated (eGFR) according to the Modification of Diet in Renal Disease (MDRD) in CKD patients and by the formula of the Mayo Clinics [19] in controls with normal renal function. Proteinuria was assessed in morning spot urine by determination of the ration of proteinuria and urinary

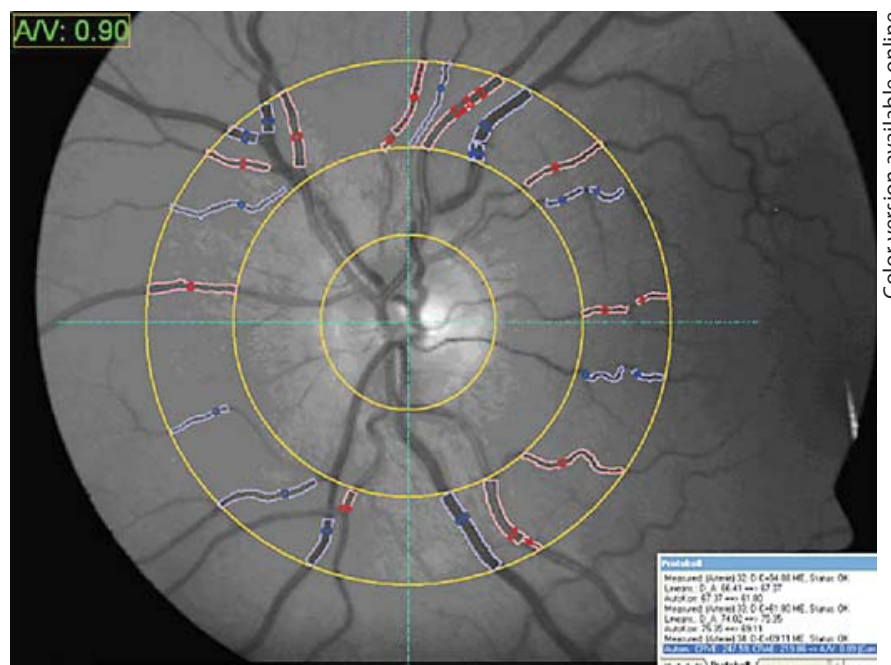

Fig. 1. Retinal photography with RVA. A/V represents AVR. Measurements are performed of all arterioles and venules in a specified zone surrounding the optic disc. Arterioles are marked in red, venules in blue (colors in the online version only).

creatinine (PCR). Cardiovascular risk factors were evaluated and biological parameters measured according to the recommendations of the American and European Clinical Practice Guidelines. The total number of antihypertensive drugs was recorded, in particular the use of calcium channel blocker and renin-angiotensin system (RAS) blocker. Diabetes mellitus was an exclusion criterion for both groups and defined as a fasting blood glucose level of at least $6.7 \mathrm{mmol} / \mathrm{l}$ or as the use of antidiabetic drugs. The inclusion criterion for the CKD group was stage $2-5$. For the volunteer group, an eGFR $<60 \mathrm{ml} / \mathrm{min} / 1.73 \mathrm{~m}^{2}$ was an exclusion criterion. Volunteers were selected to achieve an age-matched cohort.

One trained person measured anthropometric characteristics, and the body mass index was calculated. Venous blood samples, collected after overnight fasting, were analyzed by standard automated methods for lipids and blood glucose. Office BP was determined in the sitting position after a 5 -min period of rest (Omron, Tokyo, Japan). The values represent the mean of at least three measurements.

One trained person performed the retina measurements. Methods used to measure and summarize retinal vessel diameters from digitized photographs followed a standardized protocol described elsewhere [10]. In brief, two $30^{\circ}$ color retinal photographs of the left and two photographs of the right eye were taken at baseline (fig. 1). They were digitized by a high-resolution scanner with standard settings (IMEDOS, Jena, Germany). Graders masked to the characteristics of participants used a computer program to measure diameters of all arterioles and venules in a specified zone surrounding the optic disc. These measurements were combined into summary indices - the central retinal arteriolar and venular equivalents - which represented the average arteriolar and venular diameters of that eye, respectively. These were finally expressed as the retinal AVR. The ratio compensates for possible magnification differences between eyes, and an AVR of 1 indi- 

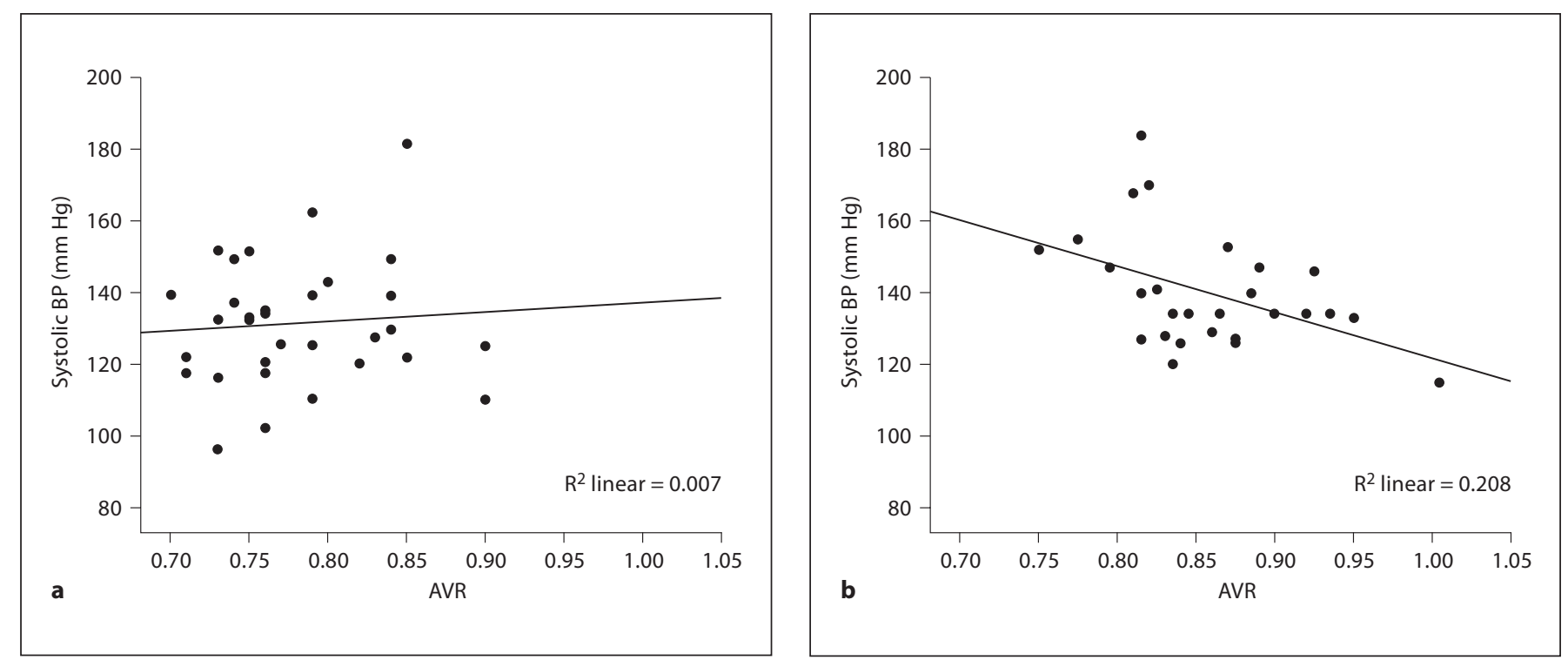

Fig. 2. Association of systolic BP and AVR in $\mathbf{a} C K D$ patients and $\mathbf{b}$ age-matched volunteers.

cates that arteriolar diameters are, on average, the same as venular diameters in that eye, while a smaller ratio suggests narrower arterioles. The AVR reflects the mean of the four photographs.

Data are expressed as mean \pm SD or percentages. The unpaired $t$ test or the Wilcoxon rank sum tests were used, respectively. Differences in frequency were tested by $\chi^{2}$ analysis. Association was determined using the Pearson coefficient. Multivariate regression analysis was performed to independent predictors for AVR. Data were analyzed using SPSS software (version 14.0). All tests were two-sided and a $p$ value of $<0.05$ was considered significant.

\section{Results}

The characteristics of the non-diabetic CKD patients and age-matched volunteers are presented in table 1 . The cause of CKD was glomerulonephritis in 15 patients, urological diseases in 9 patients and hypertensive nephropathy in 10 patients. The stages of CKD were distributed as follows: 9 patients stage 2, 15 patients stage 3, 7 patients stage 4 , and 3 patients stage 5 .

AVR was negatively correlated with systolic BP in volunteers ( $\mathrm{r}=-0.46, \mathrm{p}<0.05$; fig. 2$)$, but not with age $(\mathrm{r}=$ $0.20)$ and eGFR $(r=0.02)$. Metabolic factors had no influence on AVR. Central retinal arteriolar equivalent (CRAE) was negatively associated with systolic BP ( $\mathrm{r}=$ $-0.38)$ and diastolic BP $(r=-0.45, p<0.05)$ and positively associated with eGFR $(r=0.47, \mathrm{p}<0.05)$, but not with
Table 1. Characteristics of non-diabetic CKD patients and healthy age-matched controls

\begin{tabular}{lcc}
\hline Characteristics & $\begin{array}{l}\text { CKD } \\
\text { patients } \\
(\mathrm{n}=34)\end{array}$ & $\begin{array}{c}\text { Control } \\
\text { subjects } \\
(\mathrm{n}=33)\end{array}$ \\
\hline Age, years & $62.3 \pm 14.2$ & $61.5 \pm 10.6$ \\
Male gender, \% & 50 & 61 \\
BMI, kg/m ${ }^{2}$ & $29.4 \pm 7.7$ & $26.3 \pm 8.9$ \\
Smoking, \% & 9 & 15 \\
eGFR, ml/min/1.73 m ${ }^{2}$ & $\mathbf{4 5 . 3} \pm \mathbf{2 3 . 7}$ & $\mathbf{8 6 . 3} \pm \mathbf{9 . 1}$ \\
Protein-creatinine ratio, mg/g & $\mathbf{3 8 8} \pm \mathbf{3 4 3}$ & - \\
Glycemia, mmol/l & $5.1 \pm 0.7$ & $4.6 \pm 0.7$ \\
Cholesterol HDL, mmol/l & $\mathbf{5 5 . 8} \pm \mathbf{1 5 . 5}$ & $\mathbf{6 9 . 3} \pm \mathbf{1 3 . 8}$ \\
Cholesterol LDL, mmol/l & $128.2 \pm 50.0$ & $147.9 \pm 41.4$ \\
Systolic BP, mm Hg & $131.2 \pm 17.2$ & $138.4 \pm 17.1$ \\
Diastolic BP, mm Hg & $\mathbf{0 7 3 . 6} \pm \mathbf{1 2 . 1}$ & $\mathbf{8 4 . 0} \pm \mathbf{1 1 . 6}$ \\
Heart rate, bpm & $\mathbf{6 9 . 3} \pm \mathbf{1 3 . 4}$ & $\mathbf{5 8 . 8} \pm \mathbf{8 . 1}$ \\
Antihypertensive drugs, $\mathrm{n}$ & $\mathbf{2 . 0} \pm \mathbf{1 . 0}$ & - \\
RAS blocker, \% & $\mathbf{7 0}$ & - \\
CCB, \% & $\mathbf{4 1}$ & - \\
AVR & $\mathbf{0 . 7 7} \pm \mathbf{0 . 0 5}$ & $\mathbf{0 . 8 6} \pm \mathbf{0 . 0 6}$ \\
CRAE, $\mu \mathrm{m}$ & $\mathbf{1 6 9 . 6} \pm \mathbf{2 0 . 4}$ & $\mathbf{1 8 9 . 5} \pm \mathbf{1 4 . 2}$ \\
CRVE, $\mu \mathrm{m}$ & $\mathbf{2 1 8 . 3 \pm 2 1 . 2}$ & $\mathbf{2 2 8 . 0} \pm \mathbf{1 4 . 9}$ \\
& &
\end{tabular}

Values represent mean $\pm \mathrm{SD}$ or percentages (\%).

HDL = High-density lipoprotein; LDL = low-density lipoprotein; $\mathrm{BP}=$ blood pressure; RAS = renin-angiotensin system; $\mathrm{CCB}=$ calcium channel blocker; $\mathrm{CRAE}=$ central retinal arteriolar equivalent; $\mathrm{CRVE}=$ central retinal venular equivalent. Significance level $\mathrm{p}<0.05$, significant $\mathrm{p}$ values: bold. 


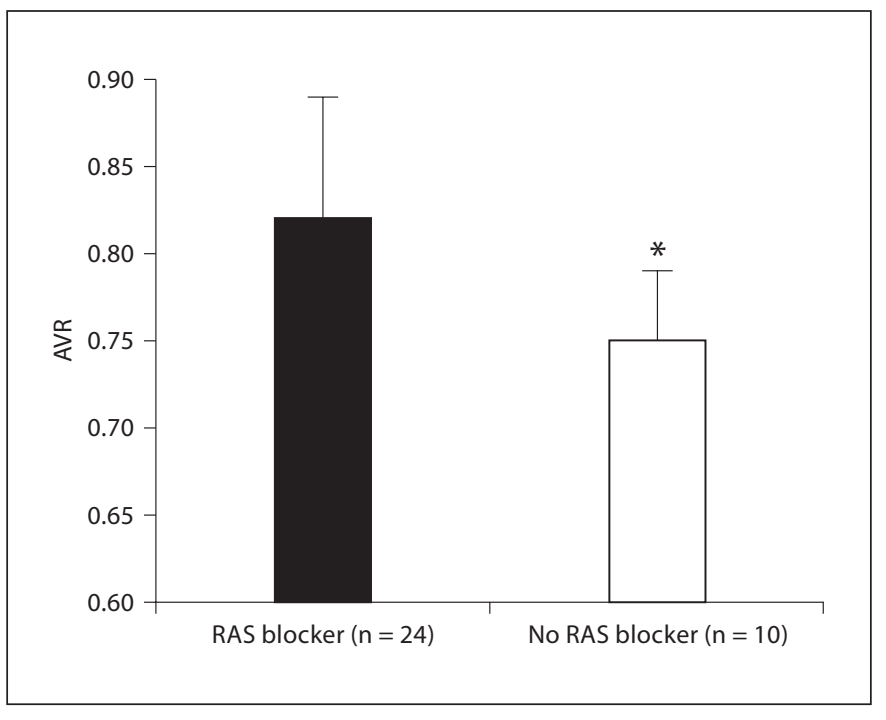

Fig. 3. AVR in CKD patients depending on RAS blockade. * $\mathrm{p}<$ 0.05 .

Table 2. Multiple regression analysis for AVR in non-diabetic CKD patients

\begin{tabular}{lrl}
\hline & $\beta$ & $\mathrm{p}$ \\
\hline Gender $(1=$ male, 2 = female $)$ & -0.13 & 0.37 \\
Age, years & 0.18 & 0.57 \\
eGFR, $\mathrm{ml} / \mathrm{min} / 1.73 \mathrm{~m}^{2}$ & 0.48 & 0.02 \\
Systolic BP, mm Hg & -0.51 & 0.01 \\
RAS blocker $(1=$ yes, $2=$ no) & 0.40 & 0.03 \\
PCR & 0.08 & 0.80 \\
\hline
\end{tabular}

$\mathrm{R}^{2}=0.30$.

Table 3. Multiple regression analysis for the independent prediction renal function (eGFR)

\begin{tabular}{lrl}
\hline & $\beta$ & $\mathrm{p}$ \\
\hline Gender $(1=$ male, 2 = female $)$ & 0.08 & 0.58 \\
Age, years & -0.39 & 0.01 \\
CRAE, $\mu \mathrm{m}$ & 0.33 & 0.02 \\
Systolic BP, mm Hg & 0.56 & 0.001 \\
RAS blocker $(1=$ yes, $2=$ no) & -0.21 & 0.19 \\
PCR & -0.19 & 0.17 \\
\hline
\end{tabular}

$\mathrm{R}^{2}=0.52$. age $(r=-0.11)$ or any metabolic factor. Central retinal venular equivalent (CRVE) was positively associated with eGFR $(\mathrm{r}=0.46, \mathrm{p}<0.05)$, but not with BP $(\mathrm{r}=0.15)$, age $(\mathrm{r}=-0.03)$ or any metabolic factor.

In non-diabetic CKD patients, AVR did not show any association with $\mathrm{BP}(\mathrm{r}=0.08$; fig. 2$)$, age $(\mathrm{r}=0.19)$ or eGFR $(r=0.03)$. RAS blockade and PCR were positively associated with AVR $(\mathrm{r}=0.44$ and $\mathrm{r}=0.29, \mathrm{p}<0.01$ and $\mathrm{p}=$ 0.08 ; fig. 3). In contrast, calcium channel blockade was not associated with AVR. Neither CRAE nor CRVE showed any correlation with BP, eGFR, PCR, age or metabolic factors (all p > 0.05).

In non-diabetic CKD patients, multiple regression analysis showed that age, systolic BP and RAS blockade independently predicted arteriolar diameter, whereas proteinuria lost its value after adding RAS blockade into the model (table 2). Additionally, retinal arteriolar diameter independently predicted eGFR $(\beta=0.33, \mathrm{p}<0.01$; table 3).

Regarding the validity of digitized retinal photographs, we performed measurement in 10 hemodialysis patients and repeated the measurement after 1 week. These measurements showed a Pearson correlation of 0.82 between the first and the second measurement.

\section{Discussion}

Retinal arterioles were narrower in non-diabetic CKD patients as compared to age-matched controls. In CKD patients, eGFR, BP and RAS blockade were independent predictors for arteriolar diameter, whereas proteinuria showed no relevance. Retinal arteriolar diameter predicted renal function.

The first major finding was that the retinal vessels were narrower in non-diabetic CKD patients as compared to age-matched controls with normal renal function, which was based on significantly smaller retinal arterioles, resulting in lower AVR in CKD patients. These data confirm epidemiological studies demonstrating an association between a decline in renal function and low AVR $[15,16]$. The latter reflects an elevated cardiovascular risk [14] and more specifically, cerebral white matter lesions [20] and the incidence of stroke [21]. Thus, low AVR reveals an elevated cerebrovascular risk in CKD patients as compared to our control group, which is in line with common sense [22]. Moreover, this suggests that the extrarenal vascular alterations in CKD patients are not restricted to large artery changes [23], but contain microvascular changes.

Kidney Blood Press Res 2009;32:428-433 
The second major finding is that in CKD patients, arteriolar diameter independently predicted eGFR, BP and RAS blockade, but not proteinuria. Epidemiological cohorts demonstrated a role of renal function in elderly subjects with declining eGFR on retinal microvascular abnormalities, suggesting that systemic microvascular disease may be associated with progressive renal dysfunction in the elderly population $[16,24]$. This is the first study documenting this relationship in CKD patients with an eGFR $\leq 60 \mathrm{ml} / \mathrm{min} / 1.73 \mathrm{~m}^{2}$.

In this line, we additionally confirm the role of $\mathrm{BP}$ as an independent predictor for arteriolar diameter in these subjects. Interestingly, this study shows for the first time that pharmacological interventions with RAS blocker result in a larger arteriolar diameter in CKD patients. We speculate that RAS blocker in this context may exert a direct effect on vascular remodeling $[25,26]$ and additionally decelerate atherosclerosis [27].

Proteinuria, the most commonly used marker for microvascular damage [28], lost its association with the ret- inal microvasculature by adding the factor of RAS blockade. This further strengthens the ideas of a direct effect of these drugs on the microcirculation which becomes visible in the retina by increased arteriolar diameter. Moreover, we demonstrate that the narrow retinal microvasculature predicts low eGFR, suggesting that the retina partially mirrors the renal microvasculature in CKD patients, which is essential for renal function. Finally, we demonstrate that retinal photography in CKD patients is a safe and reproducible measure as the intraindividual repetition of retinal photography with 1 week difference showed a correlation of $r=0.82$.

In summary, this study showed that retinal vessels of non-diabetic CKD patients are narrower than in agematched volunteers. Furthermore, age, BP and RAS blockade are independent predictors for the retinal microvasculature in non-diabetic CKD patients in this cohort. Interestingly, the retinal microvasculature additionally mirrors renal function.

\section{References}

1 Struijker-Boudier HA: Retinal microcirculation and early mechanisms of hypertension. Hypertension 2008;51:821-822.

-2 Struijker-Boudier HA, le Noble JL, Messing MW, Huijberts MS, le Noble FA, van Essen $\mathrm{H}$ : The microcirculation and hypertension. J Hypertens Suppl 1992;10:S147-S156.

$\checkmark 3$ Skov K, Mulvany MJ, Korsgaard N: Morphology of renal afferent arterioles in spontaneously hypertensive rats. Hypertension 1992;20:821-827.

$\checkmark 4$ Rizzoni D, Porteri E, Boari GE, De Ciuceis C, Sleiman I, Muiesan ML, Castellano M, Miclini M, Agabiti-Rosei E: Prognostic significance of small-artery structure in hypertension. Circulation 2003;108:2230-2235.

$\checkmark 5$ De Ciuceis C, Porteri E, Rizzoni D, Rizzardi N, Paiardi S, Boari GE, Miclini M, Zani F, Muiesan ML, Donato F, Salvetti M, Castellano M, Tiberio GA, Giulini SM, Agabiti Rosei E: Structural alterations of subcutaneous small-resistance arteries may predict major cardiovascular events in patients with hypertension. Am J Hypertens 2007;20:846852.

6 Feihl F, Liaudet L, Levy BI, Waeber B: Hypertension and microvascular remodelling. Cardiovasc Res 2008;78:274-285.

$>7$ Wong TY, Klein R, Couper DJ, Cooper LS, Shahar E, Hubbard LD, Wofford MR, Sharrett AR: Retinal microvascular abnormalities and incident stroke: the Atherosclerosis Risk in Communities Study. Lancet 2001; 358:1134-1140.
-8 Doubal FN, Hokke PE, Wardlaw JM: Retinal microvascular abnormalities and stroke: a systematic review. J Neurol Neurosurg Psychiatry 2009;80:158-165.

$\checkmark 9$ Van den Born BJ, Hulsman CA, Hoekstra JB, Schlingemann RO, van Montfrans GA: Value of routine funduscopy in patients with hypertension: systematic review. BMJ 2005 331:73.

$>10$ Wong TY, Shankar A, Klein R, Klein BE, Hubbard LD: Prospective cohort study of retinal vessel diameters and risk of hypertension. BMJ 2004;329:79.

11 Leung H, Wang JJ, Rochtchina E, Wong TY, Klein R, Mitchell P: Impact of current and past blood pressure on retinal arteriolar diameter in an older population. J Hypertens 2004;22:1543-1549.

12 Wong TY, Liew G, Tapp RJ, Schmidt MI, Wang JJ, Mitchell P, Klein R, Klein BE, Zimmet P, Shaw J: Relation between fasting glucose and retinopathy for diagnosis of diabetes: three population-based cross-sectional studies. Lancet 2008;371:736-743.

13 Wong TY, Klein R, Sharrett AR, Duncan BB, Couper DJ, Tielsch JM, Klein BE, Hubbard LD: Retinal arteriolar narrowing and risk of coronary heart disease in men and women: the Atherosclerosis Risk in Communities Study. JAMA 2002;287:1153-1159.

14 Wong TY, Knudtson MD, Klein R, Klein BE, Hubbard LD: A prospective cohort study of retinal arteriolar narrowing and mortality. Am J Epidemiol 2004;159:819-825.
15 Wong TY, Coresh J, Klein R, Muntner P, Couper DJ, Sharrett AR, Klein BE, Heiss G, Hubbard LD, Duncan BB: Retinal microvascular abnormalities and renal dysfunction: the Atherosclerosis Risk in Communities Study. J Am Soc Nephrol 2004;15:24692476

16 Sabanayagam C, Shankar A, Koh D, Chia KS, Saw SM, Lim SC, Tai ES, Wong TY: Retinal microvascular caliber and chronic kidney disease in an Asian population. Am J Epidemiol 2009;169:625-632.

17 Stenvinkel P, Carrero JJ, Axelsson J, Lindholm B, Heimburger O, Massy Z: Emerging biomarkers for evaluating cardiovascular risk in the chronic kidney disease patient: how do new pieces fit into the uremic puzzle? Clin J Am Soc Nephrol 2008;3:505-521.

18 Ohya Y, Iseki K, Iseki C, Miyagi T, Kinjo K, Takishita S: Increased pulse wave velocity is associated with low creatinine clearance and proteinuria in a screened cohort. Am J Kidney Dis 2006;47:790-797.

$>19$ Rule AD, Larson TS, Bergstralh EJ, Slezak JM, Jacobsen SJ, Cosio FG: Using serum creatinine to estimate glomerular filtration rate: accuracy in good health and in chronic kidney disease. Ann Intern Med 2004;141: 929-937.

-20 Wong TY, Klein R, Sharrett AR, Couper DJ, Klein BE, Liao DP, Hubbard LD, Mosley TH: Cerebral white matter lesions, retinopathy, and incident clinical stroke. JAMA 2002; 288:67-74. 
21 Cooper LS, Wong TY, Klein R, Sharrett AR, Bryan RN, Hubbard LD, Couper DJ, Heiss G, Sorlie PD: Retinal microvascular abnormalities and MRI-defined subclinical cerebral infarction: the Atherosclerosis Risk in Communities Study. Stroke 2006;37:82-86.

22 Bidani AK, Griffin KA: Long-term renal consequences of hypertension for normal and diseased kidneys. Curr Opin Nephrol Hypertens 2002;11:73-80.

-23 Blacher J, Guerin AP, Pannier B, Marchais SJ, Safar ME, London GM: Impact of aortic stiffness on survival in end-stage renal disease. Circulation 1999;99:2434-2439.
4 Edwards MS, Wilson DB, Craven TE, Stafford J, Fried LF, Wong TY, Klein R, Burke GL, Hansen KJ: Associations between retinal microvascular abnormalities and declining renal function in the elderly population: the Cardiovascular Health Study. Am J Kidney Dis 2005;46:214-224.

25 Neves MF, Virdis A, Schiffrin EL: Resistance artery mechanics and composition in angiotensin II-infused rats: effects of aldosterone antagonism. J Hypertens 2003;21:189-198.

26 Sharifi AM, Li JS, Endemann D, Schiffrin EL: Effects of enalapril and amlodipine on small-artery structure and composition, and on endothelial dysfunction in spontaneously hypertensive rats. J Hypertens 1998;16:457466.
7 Schiffrin EL, Lipman ML, Mann JF: Chronic kidney disease: effects on the cardiovascular system. Circulation 2007;116:85-97.

28 Burkhardt K, Schwarz S, Pan C, Stelter F, Kotliar K, Von Eynatten M, Sollinger D, Lanzl I, Heemann U, Baumann M: Myeloidrelated protein 8/14 complex describes microcirculatory alterations in patients with type 2 diabetes and nephropathy. Cardiovasc Diabetol 2009;8:10. 